\title{
Cell Lineage, Self-Renewal, and Epithelial-to-Mesenchy- mal Transition during Secondary Neurulation
}

\author{
Teruaki Kawachi, ${ }^{1,2}$ Ryosuke Tadokoro, ${ }^{1,2}$ Yoshiko Takahashi $^{1}$ \\ Department of Zoology, Graduate School of Science, Kyoto University, Kyoto, Japan \\ Faculty of Engineering, ${ }^{2}$ Okayama University of Science, Okayama, Japan
}

Secondary neurulation (SN) is a critical process to form the neural tube in the posterior region of the body including the tail. SN is distinct from the anteriorly occurring primary neurulation (PN); whereas the PN proceeds by folding an epithelial neural plate, SN precursors arise from a specified epiblast by epithelial-to-mesenchymal transition (EMT), and undergo self-renewal in the tail bud. They finally differentiate into the neural tube through mesenchymal-to-epithelial transition (MET). We here overview recent progresses in the studies of SN with a particular focus on the regulation of cell lineage, self-renewal, and EMT/MET. Cellular mechanisms underlying $\mathrm{SN}$ help to understand the functional diversity of the tail in vertebrates.

Key Words : Cell lineage · Cell self renewal · Epithelial-mesenchymal transition · Chickens.

\section{INTRODUCTION}

The tail, located posteriorly to the hind limbs, is one of the traits that characterize the vertebrates, and endows animals with a variety of locomotive functions ${ }^{13)}$. The tail is relatively of simple structure, containing ectoderm (skin and spinal cord) and mesoderm (vertebrae and skeletal muscles) but not endoderm. The gut endoderm ends at the anus that is located at the same level as the hind limbs at least in amniotes ${ }^{24)}$. Thus, the tail functions are mostly governed by skeletal musculatures and the spinal cord and its derivatives. The neural tube, an embryonic form of the spinal cord, in the tail bud develops by the process known as secondary neurulation ( $\mathrm{SN}$ ), which is distinct from the primary neurulation (PN) seen in the ante- rior part of the body ${ }^{1-4,9)}$. In the adult body, PN- and SN neural tubes are seamlessly connected functionally and morphologically (Fig. 1) ${ }^{15,20)}$. Contrasting with the extensively studied PN, which proceeds by folding the epithelial cell sheet of neural plate, the mechanisms underlying $\mathrm{SN}$ are much less understood although the first description was made more than 80 years ago ${ }^{11,18)}$. One reason is that the $\mathrm{SN}$ process is less conspicuous; $\mathrm{SN}$ precursors are mesenchymal in shape residing in the tail bud along with mesodermal precursors that are also mesenchyme ${ }^{3)}$. Since it was difficult to distinguish between SN and mesodermal precursors in the tail bud, cellular functions of SN precursors remained elusive.

Recently, the SN has increasingly attracted investigators because its failure causes spina bifida at the lumber level, which is

- Received : February 25, 2021 •Revised : April 3, 2021 •Accepted : April 7, 2021

- Address for reprints : Yoshiko Takahashi

Department of Zoology, Graduate School of Science, Kyoto University, Kitashirakawa, Sakyo-ku, Kyoto 606-8502, Japan

Tel : +81-75-753-4102, Fax : +81-75-753-4102, E-mail : yotayota@develop.zool.kyoto-u.ac.jp, ORCID : https://orcid.org/0000-0002-1596-7527

This is an Open Access article distributed under the terms of the Creative Commons Attribution Non-Commercial License (http://creativecommons.org/licenses/by-nc/4.0) which permits unrestricted non-commercial use, distribution, and reproduction in any medium, provided the original work is properly cited. 


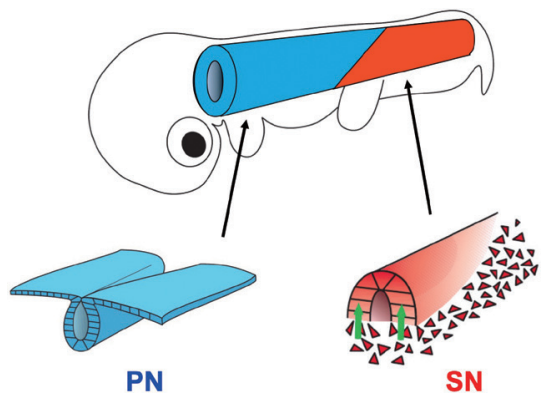

Fig. 1. Diagram to depict primary neurulation (PN; blue) and secondary neurulation ( $\mathrm{SN}$; red). Neural tubes formed by these processes are seamlessly connected in the adult body.

among the most common congenital malformations in humans ${ }^{5,16}$. In a past decade, several findings have been obtained mostly by using chicken embryos, which serve as an excellent model animal for SN studies ${ }^{5,14,22}$. We here overview some of these findings concerning the lineage of SN precursors and their participation in the neural tube formation. Dynamic behaviors of SN precursors including epithelial-to-mesenchymal transition (EMT), self-renewal, and mesenchymal-to-epithelial transition (MET) are also highlighted. We also discuss how such sophisticated neurulation emerged during vertebrate evolution.

\section{ORIGIN OF SN PRECURSORS AND EMT}

It was under big debate concerning what the early epiblast located posteriorly to the organizer ("node" in mice and "Hensen's node $[\mathrm{HN}]$ " in avians) would give rise to. In mice, this region contains the primitive streak along the midline, and its anterior end is juxtaposed to the node. Thus, the epiblastic areas located laterally to the primitive streak were called as caudolateral epiblast ${ }^{6,23,28)}$. In contrast, in chicken early embryos at Hamburger and Hamilton stage 8 (HH8; 6 somites), a conspicuous area covered by an epiblastic surface is recognized posteriorly to the $\mathrm{HN}$. Unlike mice, this area is devoid of primitive streak, which lies merely posteriorly to this area. In a report by Dady et al. ${ }^{5}$, this area was called as a node streak border (NSB) which contained PN precursors laterally and a medial region which will specifically be discussed in this article. The term of NSB is also used in mouse studies ${ }^{28)}$, but it shows a more restricted region because the node and anterior end of primitive streak are juxtaposed.

In the report by Shimokita and Takahashi ${ }^{22}$, the epiblast of chicken NSB region was scrutinized at the cellular level, particularly, for its epithelial character. Serial transverse histological sections revealed unexpected architecture: this region contains a peculiar epithelial sheet that lacks the basement membrane (BM; Laminin-positive), which would normally be associated at the basal side of the conventional epiblast epithelium. In Fig. 2, section \#13 representatively shows such epithelium without underlying BM. The white area of antero-posteriorly stretched rhomboidal shape in Fig. 2B depicts the epiblast at HH8 which is devoid of underlying BM. Since the narrow longitudinal white line covering sections \#16-\#18 shows the primitive streak known to give rise to mesoderm by EMT, the BM-free rhomboidal epiblast was predicted to undergo EMT in a similar way to the primitive streak. Indeed, local labeling of several different positions in the rhomboidal epiblast resulted in labeled cells in the tail bud at later stages ${ }^{22}$. More importantly, the labeled tail bud mesenchymal cells originated from this rhomboidal epiblast eventually participated in the neural tube formation by $\mathrm{MET}^{14,22)}$. Thus, the rhomboidal epiblast located posteriorly to $\mathrm{HN}$ at $\mathrm{HH} 8$ is a presumptive SN region (hereafter called "preSN"), which undergoes EMT to provide tail bud mesenchymal cells that subsequently differentiate into neural epithelial cells through $\mathrm{MET}^{14,22)}$. In Fig. 2B, pale red areas laterally located to the white rhomboidal region are precursors of $\mathrm{PN}^{5,22}$.

The identification of the SN precursors in early embryos immediately enabled SN-specific gene manipulation using the in ovo electroporation technique. A solution of DNA plasmid(s) successfully stays on top of the preSN because this area of epiblast is concave in shape. As expected, enhanced GFP (EGFP)electroporated preSN yields EGFP-positive mesenchymal cells in the tail bud, and ultimately in SN-neural tube ${ }^{22}$. The preSN region at $\mathrm{HH} 8$ gives rise essentially to $\mathrm{SN}$ precursors (uni-fated $\mathrm{SN}$ ), whereas mesodermal precursors reside as a minority population at the posterior edge of the rhomboid (Fig. 3) ${ }^{14)}$.

\section{SELF-RENEWAL OF SN PRECURSORS IN THE TAIL BUD}

One important question is how SN precursors are maintained in the tail bud during axial elongation. Since a decent number of SN precursors in the tail bud constantly participate in the neural tube formation by MET, this "loss" needs to be 


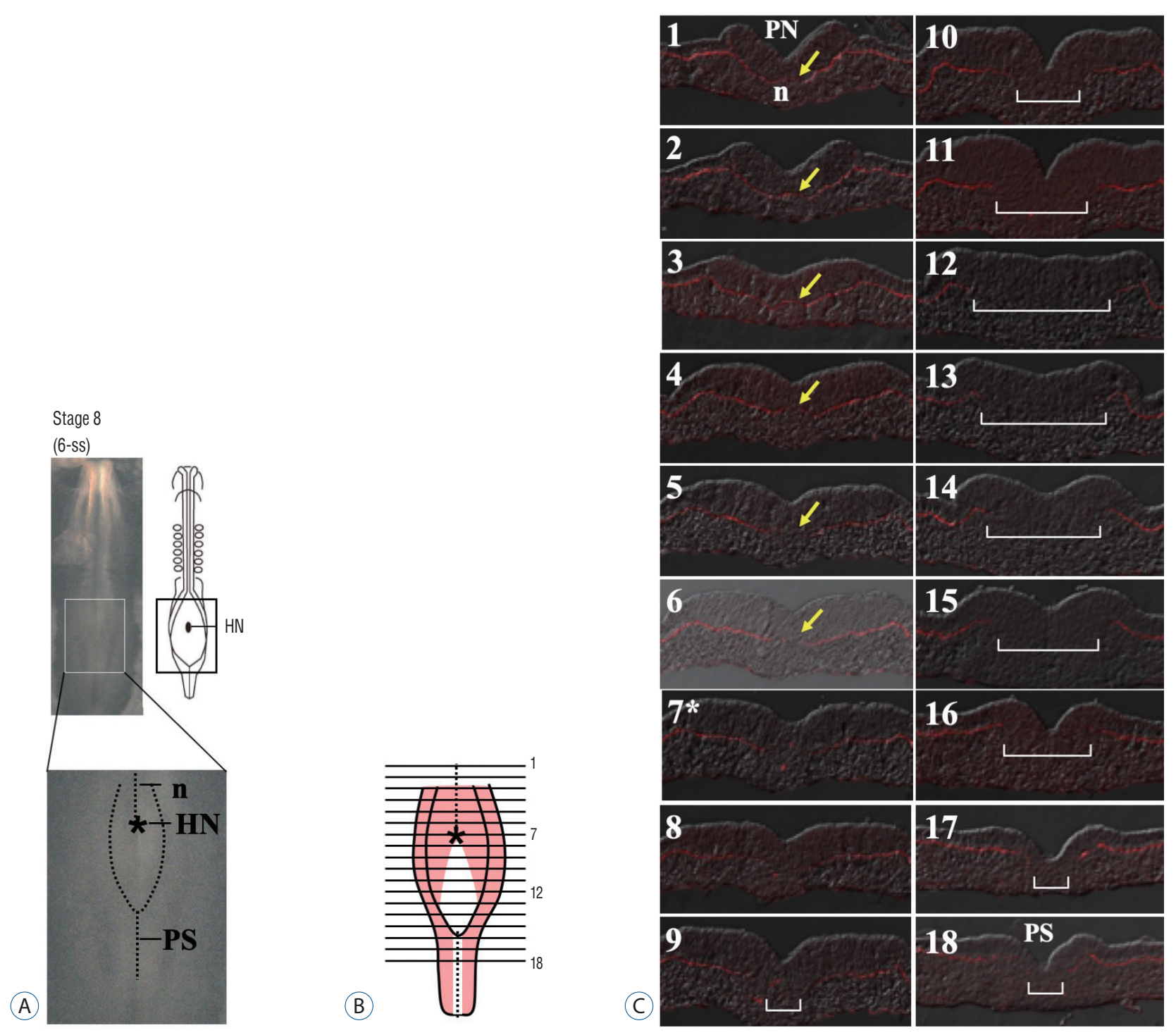

Fig. 2. $A$ and $B$ : In $\mathrm{HH} 8$ embryos, the epiblast located in between $\mathrm{HN}\left(^{*}\right)$ and the anterior end of primitive streak is devoid of underlying $B M($ white in $B$ ), whereas other epiblast areas are supported by BM (pink in B). C : Serial histological sections are stained with anti-Laminin antibody to visualize BM. White brackets indicate the epiblast lacking the underlying BM. Yellow arrows indicated notochord. Modified from Shimokita and Takahashi ${ }^{22)}$ with permnission. HN : Hensen's node, PS : primitive streak, HH8: Hamburger and Hamilton stage 8, BM : basement membrane.
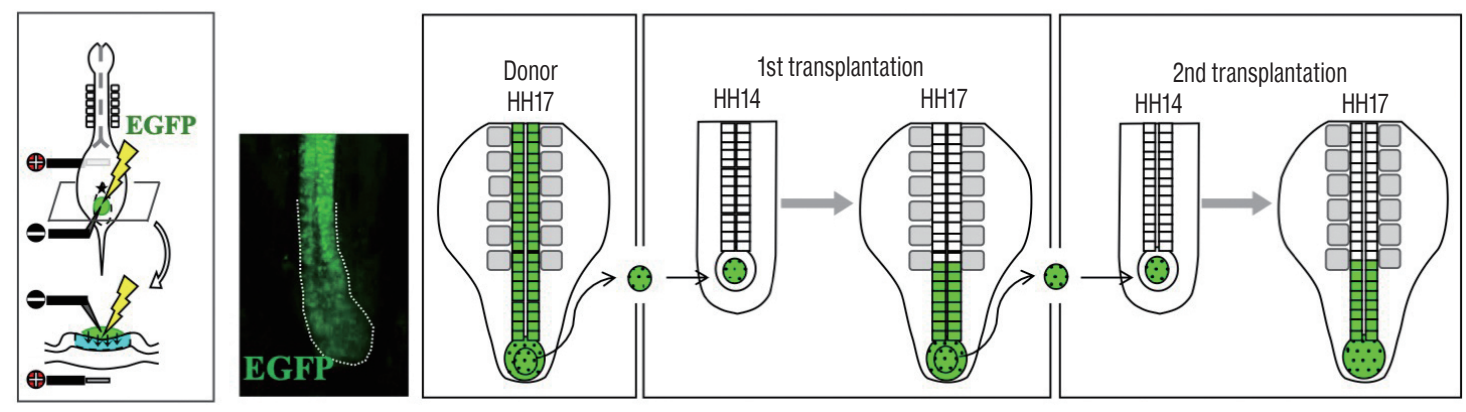

Fig. 3. Diagram showing in ovo DNA (EGFP) electroporation that targets the rhomboidal epiblast at HH8. Electroporated embryo shows EGFP-positive SN precursors in the tail bud and SN neural tube. A serial transplantation with SN precursors revealed self-renewal cells in the tail bud. Illustration was reproduced from Kawachi et al. ${ }^{14)}$ with permission. EGFP : enhanced GFP, HH: Hamburger and Hamilton, $\mathrm{SN}$ : secondary neurulation. 
compensated to maintain the tail bud. To understand how this compensation is regulated, one experiment was conducted: EGFP-labeled SN precursor cells dissected from a tail bud of donor embryo were isotopically transplanted into non-electroporated host embryos.

In the tail bud of donor embryos at HH14, which had been electroporated with EGFP DNA into the rhomboidal preSN region at $\mathrm{HH} 8$ as shown above, EGFP-labeled SN precursor cells were found in the anterior two-thirds, whereas the posterior third gave rise to mesoderm ${ }^{14}$. The finding that $\mathrm{SN}$ and mesodermal precursors are segregated in the forming tail bud gave a novel insight since the fates of tail bud-constituting cells had long been unclarified because these cells are mesenchymal that could not be distinguished by conventional histological approaches. Thus, a tracing of EGFP-electroporated cells directly in embryos proved to be a powerful technique to identify the SN precursors in embryos.

For the isotopic transplantation, EGFP-positive cells were taken from the anterior two thirds (SN territory) of tail bud of HH17 donor embryos, and implanted into an equivalent site of non-electroporated host embryos at $\mathrm{HH} 14^{14}{ }^{14}$. When assessed at HH17, the transplanted cells were, as expected, incorporated into the neural tube formation (Fig. 3). More importantly, EGFP-positive cells were also seen as mesenchymal cells in the tail bud. A serial transplantation demonstrated

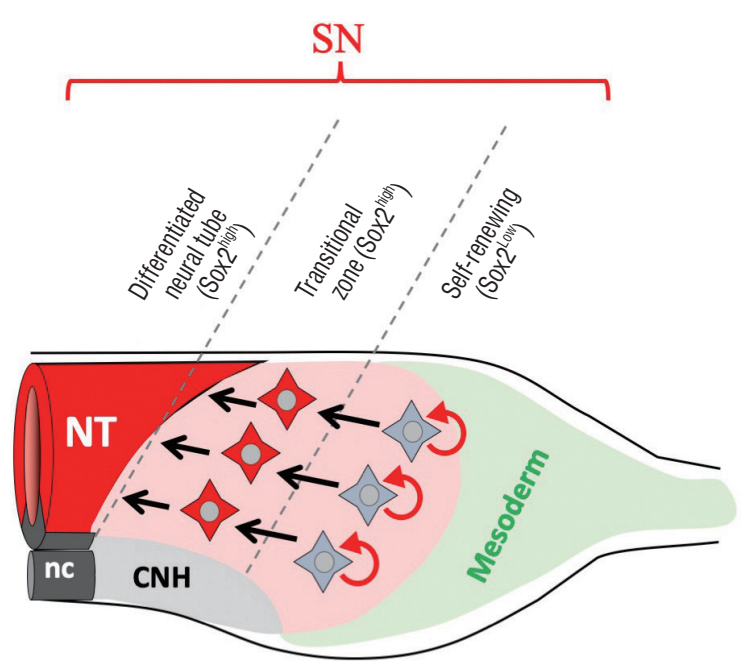

Fig. 4. Diagram showing two subtypes of SN uni-fated precursors in the tail bud at HH14. They are regulated by high and low levels of Sox 2 expression. Sox $2^{\text {high }}$ and Sox $2^{\text {low }}$ are for specification and self-renewal of SN precursors, respectively. Modified from Kawachi et al. ${ }^{14)}$ with permission. SN : secondary neurulation, NT : neural tube, nc : notochord, $\mathrm{CNH}$ : chord-neural hinge. unambiguously that the $\mathrm{SN}$ precursors in the tail bud contain self-renewing (stem cell-like) cells. The self-renewing cells were further found to be restricted to the posterior half of the SN precursor territory in the tail bud. When the anterior half of the EGFP-labeled SN territory was transplanted into a host embryo, all the EGFP-positive cells ended up in the secondary neural tube formation leaving no cells in the tail bud. Thus, these anterior cells are neural-specified cells (Fig. 4).

\section{SELF-RENEWAL OF SN PRECURSORS IS REGU- LATED BY A LOW LEVEL OF SOX2}

How is the self-renewal of SN precursors regulated? Who tells these cells to move toward the differentiation step or to remain as self-renewing cells in the tail bud? To address these questions, the role of transcriptional factor Sox 2 was investigated $^{14)}$. Sox 2 expression is generally regarded as an early marker of neural tube formation during PN since Sox 2 is already expressed in the neural plate even before the neural tube closure. During SN, however, the onset of Sox 2 occurs upon MET of SN precursors. Indeed, the rhomboidal epiblastic region in early embryos (HH8), from which SN precursors arise by EMT as explained earlier, is devoid of Sox2, whereas its adjacent PN regions (neural plate) are Sox2-positive ${ }^{26}$.

Sox 2 was found to be differentially expressed in SN precursors of the tail bud; high and low levels in the anterior and posterior halves, respectively, revealed by both in situ hybridization and immunocytochemistry. The mesodermal area in the tail bud is negative for Sox 2 . Thus, the differences in expression levels of Sox 2 are correlated with the two subtypes of SN precursors; a high level in SN-specified cells and a low level in self-renewing cells. And this correlation was further substantiated experimentally by manipulating expression levels of Sox 2 in SN precursors of the tail bud. The analyses were conducted by spatio-temporally controlled gene manipulations. For overexpression of Sox2, the Tet-on system was used ${ }^{27)}$ so that SN precursors that completed EMT to become mesenchymal cells in the tail bud were induced to overexpress Sox 2 . After 6 hours, Sox2-overexpressed cells visualized by co-expressing EGFP underwent precocious differentiation into neural epithelial cells with no self-renewing cells left in the tail bud. In contrast, Sox2-deprived cells using dominant negative form failed to undergo both self-renewal and neural differen- 
tiation. These carefully conducted gene manipulations in a spatio-temporally controlled manner allowed the investigators to conclude that the high level of Sox 2 is critical for the specification of SN precursors toward the neural differentiation by MET, and that a low level of Sox2 is also important probably for surviving of self-renewing SN precursors in the tail bud.

\section{SN UNI-FATED PRECURSORS ARE RE-DIRECT- ED TO BI-FATED TO PRODUCE NEUROMESO- DERM AT LATER STAGES}

It is until HH17 that the rhomboidal epiblast-derived cells stay as uni-fated SN precursors. When these cells (EGFP-electroporated) were traced until later stages in the same embryo, EGFP-cells were observed not only in SN-neural tube but also in mesodermal tissues such as tail somites ${ }^{14}$. Thus, the rhomboidal epiblast-derived cells remain as SN uni-fated precursors until HH17, and subsequently some of these cells (probably not all) become bi-fated. Interestingly, these fate-converted cells are observed only in the tail mesoderm and never in the trunk mesoderm. It raises an interesting possibility that the lineage of mesodermal cells is different between the trunk and tail: in the trunk, mesodermal cells are produced through the primitive streak by EMT, whereas those in the tail derive by re-direction of originally SN uni-fated precursors. This idea agrees, although partly, with the concept of neuro-mesoder- mal progenitors (NMPs) ${ }^{10,19,21)}$. A currently prevailing model of NMPs is that axial progenitor cells are neuro- and mesodermal common progenitors. However, few studies have carefully considered a possible difference in the fate and/or differentiation potential of axial progenitors along the anteroposterior axis (e.g., trunk vs. tail). Furthermore, although heterotopic transplantations or in vitro cultures have frequently been carried out to assess NMPs, these experiments merely reveal "differentiation potential" of the cells, but cannot determine their normal fate in embryos. Thus, the findings of switching from uni-fated to bi-fated during SN maturation have provided novel insight into the understanding of axial elongation, tail development, and NMPs ${ }^{14}$.

Regarding studies of NMPs, Sox2/Brachury (Bra) expression has frequently been used as a marker for NMPs. It holds true for earlier stages such as HH5, where some epiblast cells give rise to both mesoderm and neural cells ${ }^{7,8,17,28)}$. However, the aforementioned SN uni-fated cells in the tail bud at HH14 contain a significant number of Sox $2 /$ Bra double positive cells $^{14)}$. Thus, Sox $2 /$ Bra expression is not an exclusive marker for NMPs.

\section{DIVERSITY OF SN RESOURCE IN VERTEBRATES : SN UNI-FATED PRECURSORS VS. NMPS}

The characteristic structure of the rhomboidal epiblast seen

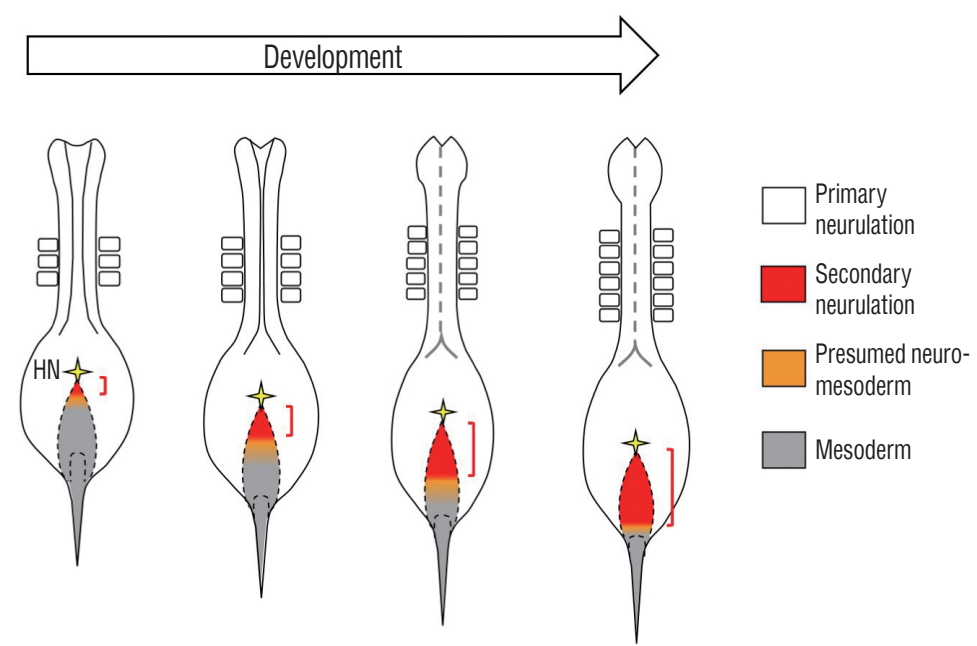

Fig. 5. Progressive expansion of the preSN domain (red) within the rhomboidal epiblast. At HH8 with 6 somites, the rhomboidal epiblast is mostly occupied by preSN. Contrary to preSN, the mesoderm-yielding domain becomes smaller. Orange areas are either NMP-producing or a mixture of neuroand mesodermal cells. preSN : presumptive SN regio, HH8: Hamburger and Hamilton stage 8, NMP : neuro-mesodermal progenitor. 
in HH8 embryos (6 somites) might be specific to avian embryos, since no such epiblast has been reported for mice. We have recently examined earlier stages such as embryos with 5 , 4 , or 3 somites. A similar rhomboidal structure of epiblast is recognized for all these stages, and cells are traced by local dye-labelling of epiblast as repeatedly explained in this article. Whereas rhomboidal epiblasts of all these stages give rise to uni-fated SN precursors, a relative portion of preSN within the epiblast is different. In young embryos with 3 somites, only a small domain adjacent to HN gives rise to $\mathrm{SN}$ uni-fated cells with the rest of the surface yielding uni-fated mesoderm or a mixture of neuro and mesoderm (or NMPs). As development proceeds, the preSN portion becomes progressively larger with HH8 embryos having the greatest occupation (Fig. 5).

It was reported using early mouse embryos that the anterior part of the NSB gives rise mostly to neural uni-fated cells, and its more posterior region yields NMPs and mesoderm ${ }^{28)}$. However, in mice, the anterior end of the primitive streak is always adjacent to the node, which is very different from the way observed in chicken embryos, in which the primitive streak and HN are intervened with a large area of primitive streak-free rhomboidal epiblast. Thus, it appears unlikely that the progressive expansion of preSN within the rhomboidal epiblast is conserved between avians and mammals. In mice, it is probable that only a small number of SN uni-fated precursors arise, and this is somehow compensated by NMPs that serve as a source of self-renewing cells for axial progenitors $^{25,28)}$. The predominant NMPs in mice might also be reflected to that the regressing primitive streak remains for a prolonged period of time, which can continuously supply axial progenitors to make a long tail. In contrast, in chickens, the primitive streak, which gives rise only to mesoderm, disappears at an earlier stage than in mice, and this is soon followed by the tail bud formation, in which SN precursors and mesodermal precursors undergo self-renewal as separate populations ${ }^{12,14)}$. NMPs in the chicken tail bud, if any at all, must be a minor population $^{14)}$. Collectively, the diversity in the SN process in chicken and mice (and other amniotes) is attributed, at least partly, to whether the expanded primitive streak-free rhomboidal epiblast is present or not at early stages, leading to preferential usage of SN uni-fated precursors or NMPs, respectively.

\section{CONCLUSION AND PERSPECTIVES}

The cellular and molecular studies of SN have begun recently. Many important questions remain unanswered. How is the rhomboidal epiblast specified to be distinct from the neighboring PN? How does the rhomboidal epiblast undergo EMT? What regulates the differential expression levels of Sox 2 in the tail bud? What determines the SN and mesoderm territories in the tail bud? How is the MET process regulated when mesenchymal SN precursors are incorporated into the neural tube? During vertebrate evolution, the emergence of the SNproducing epiblast was a big innovation because it enabled the formation of the tail neural tube, which endowed the vertebrates with an enormous diversity in life style. One such diversity is that $\mathrm{SN}$-derived spinal cord provides autonomic nervous system that governs functions of sexual organs, bladder and colon/rectum. These organs are highly divergent in physiology among vertebrates, and in particular, they acquired sophisticated functions in amniotes compared to aquatic animals. Exploration of the mechanisms underlying the SN must help to understand the diversity in the tail function during animal evolution.

\section{CONFLICTS OF INTEREST}

No potential conflict of interest relevant to this article was reported.

\section{INFORMED CONSENT}

This type of study does not require informed consent.

\section{AUTHOR CONTRIBUTIONS}

\author{
Conceptualization : TK, RT, YT \\ Writing - original draft : TK, RT, YT \\ Writing - review \& editing : TK, RT, YT
}




\section{ORCID}

Teruaki Kawachi https://orcid.org/0000-0002-6908-7031

Ryosuke Tadokoro https://orcid.org/0000-0001-7556-4263

Yoshiko Takahashi https://orcid.org/0000-0002-1596-7527

\section{- Acknowledgements}

This work was supported by JSPS KAKENHI Grant-in-Aid for Scientific Researches (B) (16KT0071) (YT), and (B) (20H03259) (YT).

\section{References}

1. Catala M : Genetic control of caudal development. Clin Genet 61 : 89 96, 2002

2. Catala M, Teillet MA, Le Douarin NM : Organization and development of the tail bud analyzed with the quail-chick chimaera system. Mech Dev $51:$ 51-65, 1995

3. Colas JF, Schoenwolf GC : Towards a cellular and molecular understanding of neurulation. Dev Dyn 221 : 117-145, 2001

4. Criley BB : Analysis of embryonic sources and mechanims of development of posterior levels of chick neural tubes. J Morphol 128 : 465501, 1969

5. Dady A, Havis E, Escriou V, Catala M, Duband JL : Junctional neurulation: a unique developmental program shaping a discrete region of the spinal cord highly susceptible to neural tube defects. J Neurosci 34 : 13208-13221, 2014

6. Garcia-Martinez V, Darnell DK, Lopez-Sanchez C, Sosic D, Olson EN, Schoenwolf GC : State of commitment of prospective neural plate and prospective mesoderm in late gastrula/early neurula stages of avian embryos. Dev Biol 181 : 102-115, 1997

7. Gouti M, Delile J, Stamataki D, Wymeersch FJ, Huang Y, Kleinjung J, et al. : A gene regulatory network balances neural and mesoderm specification during vertebrate trunk development. Dev Cell 41 : 243-261.e7, 2017

8. Gouti M, Tsakiridis A, Wymeersch FJ, Huang Y, Kleinjung J, Wilson V, et al. : In vitro generation of neuromesodermal progenitors reveals distinct roles for wnt signalling in the specification of spinal cord and paraxial mesoderm identity. PLoS Biol 12 : e1001937, 2014

9. Griffith CM, Wiley MJ, Sanders EJ : The vertebrate tail bud: three germ layers from one tissue. Anat Embryol (Berl) 185 : 101-113, 1992

10. Guillot $C$, Michaut $A$, Rabe $B$, Pourquié 0 : Dynamics of primitive streak regression controls the fate of neuro-mesodermal progenitors in the chicken embryo. bioRxiv, 2020 [Epub ahead of print]

11. Holmdahl DE : Die Morphogenese des Vertebratorganismus vom formalen und experimentellen Gesichtspunkt. W Roux' Archiv f Entwicklungsmechanik 139 : 191-226, 1939

12. limura T, Pourquié 0 : Collinear activation of Hoxb genes during gas- trulation is linked to mesoderm cell ingression. Nature $442:$ 568-571, 2006

13. Kardong KV : Vertebrates: Comparative Anatomy, Function, Evolution, 4th ed. New York: McGraw-Hill College, 2005

14. Kawachi T, Shimokita E, Kudo R, Tadokoro R, Takahashi Y : Neural-fated self-renewing cells regulated by Sox 2 during secondary neurulation in chicken tail bud. Dev Biol 461 : 160-171, 2020

15. Nievelstein RA, Hartwig NG, Vermeij-Keers C, Valk J : Embryonic development of the mammalian caudal neural tube. Teratology $48: 21-31,1993$

16. Nikolopoulou E, Galea GL, Rolo A, Greene ND, Copp AJ : Neural tube closure: cellular, molecular and biomechanical mechanisms. Development $144:$ 552-566, 2017

17. Olivera-Martinez I, Harada H, Halley PA, Storey KG : Loss of FGF-dependent mesoderm identity and rise of endogenous retinoid signalling determine cessation of body axis elongation. PLoS Biol 10 : e1001415, 2012

18. Pasteels J : Etudes sur la gastrulation des vertébrés méroblastiques. III. Oiseaux. IV Conclusions générales. Arch Biol 48 : 381-488, 1937

19. Romanos M, Allio G, Combres L, Médevielle F, Escalas N, Soula C, et al. : Cell-to-cell heterogeneity in Sox2 and Brachyury expression ratios guides progenitor destiny by controlling their motility. bioRxiv, 2020 [Epub ahead of print]

20. Saitsu H, Yamada S, Uwabe C, Ishibashi M, Shiota K : Development of the posterior neural tube in human embryos. Anat Embryol (Berl) 209 : 107-117, 2004

21. Shaker MR, Lee JH, Kim KH, Kim JV, Kim JY, Lee JY, et al. : Spatiotemporal contribution of neuromesodermal progenitor-derived neural cells in the elongation of developing mouse spinal cord. bioRxiv, 2020 [Epub ahead of print]

22. Shimokita E, Takahashi Y : Secondary neurulation: fate-mapping and gene manipulation of the neural tube in tail bud. Dev Growth Differ 53 : 401-410, 2011

23. Takemoto T, Uchikawa M, Yoshida M, Bell DM, Lovell-Badge R, Papaioannou VE, et al. : Tbx6-dependent Sox2 regulation determines neural or mesodermal fate in axial stem cells. Nature $470: 394-398,2011$

24. Tucker AS, Slack JM : Tail bud determination in the vertebrate embryo. Curr Biol 5 : 807-813, 1995

25. Tzouanacou E, Wegener A, Wymeersch FJ, Wilson V, Nicolas JF : Redefining the progression of lineage segregations during mammalian embryogenesis by clonal analysis. Dev Cell 17 : 365-376, 2009

26. Uchikawa M, Yoshida M, Iwafuchi-Doi M, Matsuda K, Ishida Y, Takemoto $T$, et al. : B1 and B2 Sox gene expression during neural plate development in chicken and mouse embryos: universal versus speciesdependent features. Dev Growth Differ 53 : 761-771, 2011

27. Watanabe T, Saito D, Tanabe K, Suetsugu R, Nakaya Y, Nakagawa S, et al. : Tet-on inducible system combined with in ovo electroporation dissects multiple roles of genes in somitogenesis of chicken embryos. Dev Biol 305 : 625-636, 2007

28. Wymeersch FJ, Huang Y, Blin G, Cambray N, Wilkie R, Wong FC, et al. : Position-dependent plasticity of distinct progenitor types in the primitive streak. Elife 5 : e10042, 2016 